the analogue of the procedure. We consider the $(n-1)$-dimensional spaces $V_{k i}$ $(i=1,2, \cdots, n)$ through $O$, whose equations are $\sum a_{i k} x_{i}=0$. When we take arbitrary values for the unknown quantities and correct them in the order $x_{k_{1}}, x_{k_{2}}, \cdots$, the construction runs as follows. An arbitrary point $P_{0}$ is chosen in $n$-space; $P_{1}$ is the orthogonal projection of $P_{0}$ on $V_{k_{1}} ; P_{2}$ is the projection of $P_{1}$ on $V_{k_{2}}$, etc. And so the procedure is illustrated in a simple geometrical way. We add some supplementary remarks. The point $P_{m}(m>0)$ lies always in one of the spaces $V_{k}$. These spaces are linearly independent if $\left|a_{i k}\right| \neq 0$. At each step (after the first) one of the $V_{k i}$ is projected by parallel projection onto the following one. In this way, an affine correspondence is established between the two successive $V_{k_{i}}$, the modulus of the affinity being $\cos \alpha$, where $\alpha$ is the angle between them. Thus the convergence of the procedure can easily be proved, provided that the corrections take place in a fixed cyclic order $x_{k_{1}}, x_{k_{2}}, \cdots$, $x_{k_{n}}$ where $k_{1}, k_{2}, \cdots, k_{n}$ is a permutation of $1,2, \cdots, n$.

If two successive $V_{k_{i}}$ are perpendicular to each other, the projection of the first onto the second coincides with their $(n-2)$-dimensional space of intersection. It follows, therefore, that if all the $V_{k i}$ are mutually perpendicular (that is, if the matrix $\left\|a_{i k}\right\|$ is orthogonal), the point $P_{1}$ lies on $V_{k_{1}}, P_{2}$ on the intersection of $V_{k_{1}}$ and $V_{k_{2}}$, $P_{3}$ on $\left(V_{k_{2}}, V_{k_{2}}, V_{k_{3}}\right)$ and so on; hence $P_{n}$ coincides with $O$. In this case the procedure ends automatically after $n$ steps.

\title{
A SIMPLIFIED METHOD OF DIFFERENTIATING AND EVALUATING FUNCTIONS REPRESENTED BY FOURIER SERIES*
}

\section{By A. M. WINSLOW (University of Washington)}

1. Introduction. This paper shows how to eliminate the difficulties caused by discontinuities of Fourier sine series at the ends of the interval of periodicity.

Applications of Fourier series to exact solutions of problems in mathematical physics involve the following essential considerations. In an interval $-a \leqq x \leqq a$, it is assumed that a function $f(x)$ and its successive derivatives up to some finite order $f^{(m)}(x)$ all comply with sufficient conditions of continuity, bounded variation, differentiability and integrability. They thus permit representation by Fourier series, which can be differentiated to give the derivative of next higher order, and integrated to give an expression for the derivative of next lower order.

When $f(x)$ is an $x$-odd function, and $f(a) \neq f(-a) \neq 0$, particular difficulties are encountered. The corresponding Fourier sine series is discontinuous at $x= \pm a$ and does not conveniently represent the values of $f(a)$ and $f(-a)$. In addition, the derivative $f^{\prime}(x)$ is represented by a complicated Fourier series which is not readily evaluated at $x= \pm a$. Thus

$$
f(x)=\sum_{1}^{\infty} b_{n}^{\prime} \sin \beta_{n} x,
$$

in which $\beta_{n}=n \pi / a$. The expression for the derivative is

*Received March 28, 1949. 


$$
f^{\prime}(x)=\frac{1}{2 a}[f(a)-f(-a)]+\sum_{1}^{\infty}\left\{{\frac{(-1)^{n}}{a}}^{n}[f(a)-f(-a)]+b_{n}^{\prime} \beta_{n}\right\} \cos \beta_{n} x .
$$

2. Modification of Fourier sine series. These difficulties of evaluation and differentiation are alleviated by rewriting Eq. (1) in the form

$$
f(x)=c x+\left(\sum_{1}^{\infty} b_{n}^{\prime} \sin \beta_{n} x-c x\right),
$$

where $c=f(a) / a$. The last term of Eq. (3) may be written

$$
c x=\sum_{1}^{\infty} b_{n}^{\prime \prime} \sin \beta_{n} x
$$

then

$$
\sum_{1}^{\infty} b_{n}^{\prime} \sin \beta_{n} x-c x=\sum_{1}^{\infty} b_{n} \sin \beta_{n} x
$$

in which $b_{n}=b_{n}^{\prime}-b_{n}^{\prime \prime}$.

In Eq. (4), $\sum_{1}^{\infty} b_{n} \sin \beta_{n} x$ represents a function having the value zero at $x= \pm a$. It consequently permits termwise differentiation.

Thus the original equation (1) is now

$$
f(x)=c x+\sum_{1}^{\infty} b_{n} \sin \beta_{n} x .
$$

At $x= \pm a, f(x)= \pm c a$, respectively. Also the derivative $f^{\prime}(x)$, correctly obtained by termwise differentiation, is

$$
f^{\prime}(x)=c+\sum_{1}^{\infty} a_{n} \cos \beta_{n} x
$$

where $a_{n}=b_{n} \beta_{n}$.

In this manner, with complete generality, Eqs. (1) and (2) can always be rewritten in the form of Eqs. (1a) and (2a).

Furthermore, in the case of integration of an $x$-even function $f^{\prime}(x)$ expressed by Eq. (2a), termwise integration is a legitimate process. If it is known that the indefinite integral $f(x)$ is an $x$-odd function, there is no additional constant of integration, and $f(x)$ is expressed by Eq. (1a), in which $b_{n}=a_{n} / \beta_{n}$. Also at $x= \pm a, f(x)= \pm c a$, respectively. This follows from the fact that termwise differentiation of Eq. (1a) gives Eq. (2a). Therefore, $\sum_{1}^{\infty} b_{n} \sin \beta_{n} x$, complying with the necessary condition of termwise differentiation, must represent a function having the value zero at $x= \pm a$.

3. Derivatives of any finite order. More general results for a mixed function are obtained by successive integrations of $f^{(m)}(x)$ with introduction of constants of integration. Thus

$$
f^{(m)}(x)=c^{\prime}+\sum_{1}^{\infty}\left(a_{n}^{\prime} \cos \beta_{n} x+b_{n}^{\prime} \sin \beta_{n} x\right)
$$

after $m$ successive integrations gives an expression of the form

$$
f(x)=\sum_{0}^{m} c_{n} x^{n}+\sum_{1}^{\infty}\left(a_{n} \cos \beta_{n} x+b_{n} \sin \beta_{n} x\right) .
$$


Conversely, derivatives up to the order $f^{(m)}(x)$ may be obtained from Eq. (6) by termwise differentiation. In the expressions for $f(x)$ and any derivative up to $f^{(m-1)}(x)$, any sine series represents a function having the value zero at $x= \pm a$, thus facilitating evaluation at these points. In some solutions Eq. (5) is a convenient expression for $f^{(m)}(x)$. If desired, Eq. (5) can be modified by adding a term $c^{\prime \prime} x$ combined with $\sum_{1}^{\infty}$ $b_{n}^{\prime} \sin \beta_{n} x$ so that the sine series in this equation also represents a function having the value zero at $x= \pm a$. Then Eq. (6), modified by this additional term, is

$$
f(x)=\sum_{0}^{m+1} c_{n} x^{n}+\sum_{1}^{\infty}\left(a_{n} \cos \beta_{n} x+b_{n} \sin \beta_{n} x\right) .
$$

Equations (6) and (6a) are special cases of Borel's theorem ${ }^{1}$ which apply to derivatives of any finite order up to $f^{(m)}(x)$.

The preceding methods of derivation, stated in terms of a single variable $x$, also apply completely to a three-dimensional member bounded by parallel planes $x= \pm a$ with assigned boundary conditions. In this case $f(x)$ is replaced by $f(x, y, z)$ and the derivatives are written as partial derivatives with respect to $x, f^{(m)}(x)$ becoming $\partial^{m} f / \partial x^{m}$. Also in Eqs. (1a), (2a), (6), (6a), every coefficient $a_{n}, b_{n}, c_{n}, c$, while independent of $x$, is a function of $y$ and $z$. For example, in Eq. (1a), $b_{n}$ is $b_{n}(y, z)$, and $c$ is $c(y, z)=$ $f(a, y, z) / a$. Thus $f(x, y, z)$ and each of its $x$-derivatives of finite order can be expressed as the sum of a finite power series and a Fourier series without discontinuity at $x= \pm a$; the expressions can, therefore, be evaluated definitely at these boundaries.

${ }^{1} \mathrm{E}$. Borel, Lȩons sur les fonctions de variables réelles, Gauthier-Villars, Paris, 1905, p. 68.

\section{A TAPERED LINE TERMINATION AT MICROWAVES*}

\section{By GEORGE J. CLEMENS (City College of New York)}

1. Introduction. In the field of ultra-high frequency, one method of power transmission is by means of concentric metallic conductors called a co-axial transmission line. The maximum amount of power can be delivered over these lines if the impedance of the load is equal to the characteristic impedance ${ }^{1}$ of the line. In addition, in various measurements at ultra-high frequency it is essential to have a matched termination over a broad band of frequencies. This problem was approached experimentally and led to the tapered line termination as shown in Fig. 1.

To the left of $x=0$, the co-axial line has an inner metallic conductor of radius $a$. From $x=0$ to $x=L$, the inner conductor is a glass tube coated with a thin metallic film of resistive material. ${ }^{2}$ To the left of $x=0$, the outer conductor has a radius $b$ and from $x=0$ to $x=L$, the outer conductor has a linear taper down to a radius $c$. Also at $x=L$, the inner and outer conductors are short circuited.

*Received Dec. 2, 1948. The results and general method of this paper conform to a section of the author's doctoral thesis, the research for which was carried on under the direction of Dr. Ernst Weber at the Polytechnic Institute of Brooklyn.

1J. C. Slater, Microwave transmission, McGraw Hill, 1942, pp. 71-74.

${ }^{2}$ Publication P. B. 6588 (U. S. Commerce Dept.), 1945. 